\title{
Laparoscopic liver hanging maneuver through the retrohepatic tunnel on the right side of the inferior vena cava combined with a simple vascular occlusion technique for laparoscopic right hemihepatectomy
}

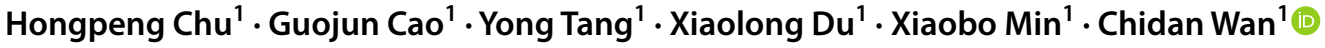

Received: 21 May 2017 / Accepted: 6 December 2017 / Published online: 21 December 2017

(c) The Author(s) 2017. This article is an open access publication

\begin{abstract}
Background Laparoscopic hepatectomy has been performed in many hospitals, with the development of the laparoscopic operation technique. However, performing complex laparoscopic hepatectomy, such as right hemihepatectomy, is still a challenge. The aim of this study was to describe the application of a simple vascular occlusion technique and new liver hanging maneuver (LHM) in complex laparoscopic hepatectomy, which are both advocated by Chen Xiaoping for open hepatectomy. Methods The clinical data of 29 consecutive patients who underwent laparoscopic right hemihepatectomy (LRH) from October 2014 to October 2016 were retrospectively analyzed. During operation, the vascular occlusion technique without hilus dissection and LHM through the retrohepatic avascular tunnel on the right side of the inferior vena cava were used.

Result All 29 operations were successfully performed laparoscopically, while adopting Chen's methods. The study consisted of 23 patients with hepatocellular carcinoma, four patients with intrahepatic cholangiocarcinoma, and two patients with hepatic metastasis of colonic carcinoma. The tumor size was $12.4 \pm 1.9 \mathrm{~cm}$. The operation time of LRH was $190.3 \pm 49.9 \mathrm{~min}$. The intraoperative blood loss of LRH was $281.7 \pm 117.8 \mathrm{~mL}$; five patients required blood transfusion, and the amount of blood transfusion was $300.0 \pm 89.4 \mathrm{~mL}$. No case was converted to open surgery, and no death occurred. All resulted in R0 resections. The median free margin was $20.1 \pm 10.8 \mathrm{~mm}$. The time of postoperative oral diet intake was $2.10 \pm 0.96$ days. The complication rate was $17.2 \%$. The average hospital stay after operation was $10.0 \pm 2.9$ days.

Conclusion Complex hepatectomy is a bloodless procedure that can be performed under a laparoscope safely using Chen's methods of vascular occlusion technique and LHM.
\end{abstract}

Keywords Laparoscopy $\cdot$ Hepatectomy $\cdot$ Hemihepatectomy $\cdot$ Vascular occlusion $\cdot$ Liver hanging maneuver $\cdot$ Goldfinger Dissector

Liver resection is the main treatment option for hepatic carcinoma. Laparoscopic liver resection (LLR) could yield the same therapeutic effects with microincision, less trauma, less pain, and quicker recovery [1-3]. More LLRs were performed with the application of advanced techniques and instruments in LLR since the first LLR was reported by Reich in 1991. Laparoscopic left lateral sectionectomy has been regarded as a standard treatment option [4-6]. However, laparoscopy is not widely accepted for liver resections,

Chidan Wan

wcdwhxh@163.com

1 Department of Hepatobiliary Surgery, Union Hospital, Tongji Medical College, Huazhong University of Science and Technology, Wuhan 430022, China especially in complex hepatectomies, such as right hemihepatectomy, because of the difficulty associated with controlling bleeding and exploring the deeper region of the liver. Because of these difficulties, LRH has not still become a standard treatment option.

During open hepatectomy, Chen Xiaoping, a Chinese professor, devised a simple vascular occlusion technique and a new LHM $[7,8]$ different from the traditional LHM advocated by Belghiti et al. [9, 10] for controlling bleeding and exploring structures, which yielded good effects. This vascular occlusion technique ligated the right hemihepatic pedicle (RHP) en masse rather than performed the ligation of the vessels and bile ducts, respectively. Further, this hanging maneuver, which could allow exploration and bleeding control from the hepatic transection plane, can be safely and easily implemented, since the retrohepatic tunnel 
on the right side of IVC is avascular. It should be suited to LRH theoretically because of its simplicity and usefulness. By applying these techniques in LRH, we performed these operations successfully.

\section{Materials and methods}

\section{Patients}

Between October 2014 to October 2016, 29 consecutive patients with malignant tumors underwent LRH at the Hepatobiliary Surgery Center, Union Hospital, Tongji Medical College, Huazhong University of Science and Technology, China. Preoperative imaging and laboratory examinations were performed for the assessment of tumor size and location and liver function and operation planning, which included computer tomography (CT) and/or magnetic resonance imaging (MRI); three-dimensional reconstruction; hepatitis $\mathrm{B}$ and $\mathrm{C}$ virus serology; test for tumor markers, including alpha fetoprotein (AFP), carcinoembryonic antigen (CEA), and carbohydrate antigen 19-9 (CA19-9); and other routine biochemical tests. Preoperative decision-making for LRH was based on tumor size and location and future liver volume (FLV)/standard liver volume (SLV) calculated by three-dimensional reconstruction.

The Ethics Review Board of Wuhan Union Hospital approved this study.

\section{Selection criteria}

The selection criteria were as follows: (1) single tumor located at more than two hepatic segments of V, VI, VII, VIII without intrahepatic metastasis; (2) no tumor invasion in the first and second hepatic portals and IVC; (3) at least a Child-Pugh B level of liver function; (4) less than 15\% indocyanine green 15 min-retention rate (ICG R15); (5) no history of abdominal surgery; and (6) no serious organ damage.

\section{LRH procedures}

We used five trocars for the operations. Their puncture locations are shown in Fig. 1.

Operating procedure was as follows: (1) The teres ligament, falciform ligament, and right coronary ligament were dissected $3-5 \mathrm{~cm}$. Intraoperative ultrasound was performed routinely to ensure that no metastases were found in the future liver remnant and enough resection margin. (2) The gallbladder was routinely excised. (3) A tape was placed around the duodenohepatic ligament and the infrahepatic IVC, respectively, to ligate the first porta hepatis and IVC for bleeding control, if necessary. (4) The RHP was ligated by using Chen's vascular occlusion technique (Table 1; Fig. 2). (5) The LMH was built (Table 2; Fig. 3). (6) The plane of liver transection was determined by the ischemia line and intraoperative ultrasound and guided by the hanging tape. The Endo Gia stapler was used for dividing the RHP and right hepatic vein during transection of the hepatic parenchyma. (7) The hepatorenal, right coronary, and triangular ligaments were mobilized and divided. (8) The part of the liver to be removed was extracted via an $8-\mathrm{cm}$ suprapubic incision.

\section{Statistical analysis}

All analyses were performed using SPSS 17.0 software. Numerical data were expressed as $\bar{x} \pm \mathrm{s}$.
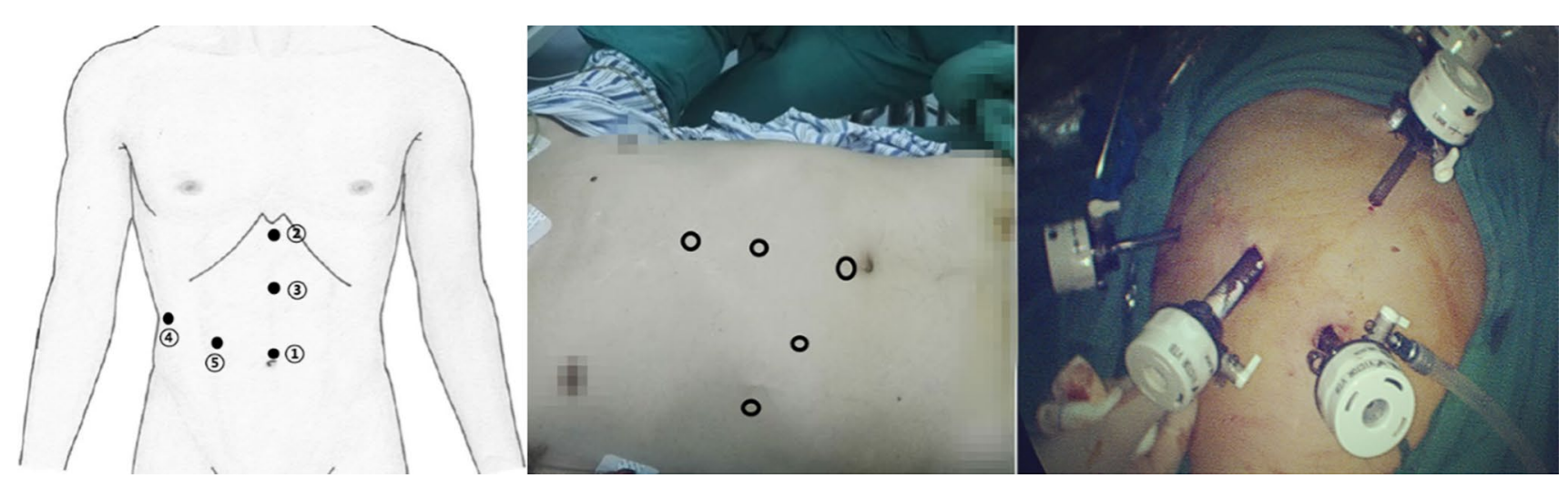

Fig. 1 Trocar sites. (1) Above the navel (10 mm); (2) below the xiphoid process (12 $\mathrm{mm})$; (3) between the previously mentioned two locations $(5 \mathrm{~mm})$; (4) below the right rib margin and along anterior axillary line $(5 \mathrm{~mm})$; (5) between first and fourth location $(12 \mathrm{~mm})$ 
Table 1 Steps of ligating the right hemihepatic pedicle by Chen's vascular occlusion technique

Order Steps
(1) Inserting a flat and cambered Goldfinger Dissector into the hepatic parenchyma $2-3 \mathrm{~cm}$ without dissecting the connective tissue on the
surface of the hilar transverse fissure at the right base of segment IV, about $1.0-1.5 \mathrm{~cm}$ right of the margin of the gastroduodenal liga-
ment
Overriding the Glisson's sheath of the right hemihepatic pedicle, then guiding the dissector towards the right posteroinferior paren-
chyma, finally penetrating the parenchyma at the caudate process of the right inferior margin of the hilar transverse fissure
A no.0 suture is pulled through the tunnel by the Goldfinger Dissector. Then ligating the right hemihepatic pedicle en masse rather than
performing ligation of the vessels and bile ducts, respectively
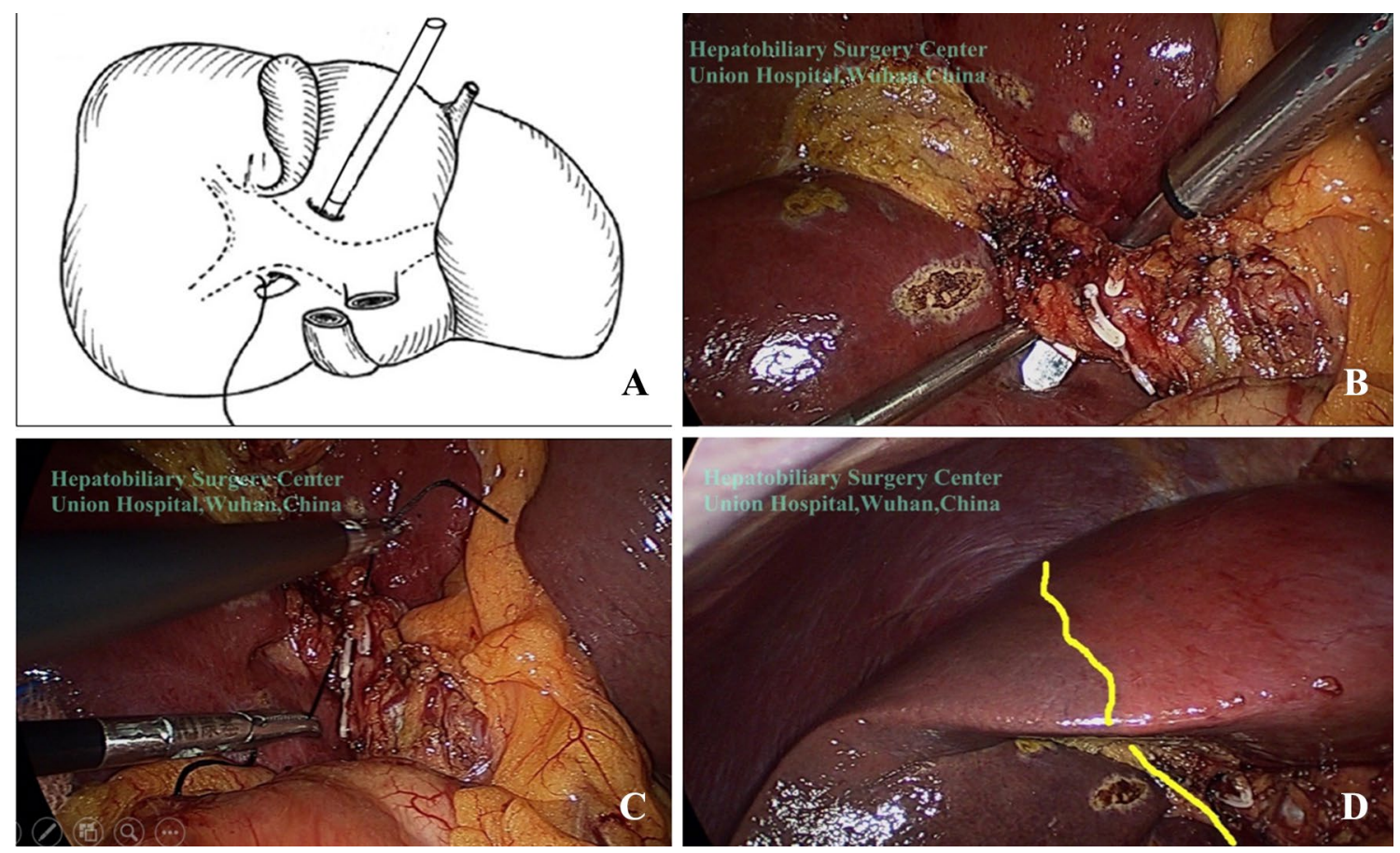

Fig. 2 Ligating the RHP. A, B Insert a flat and cambered Goldfinger Dissector into the hepatic parenchyma Overriding the Glisson's sheath of the right hemihepatic pedicle; C A No.0 suture is pulled

through the tunnel to ligate the right hemihepatic pedicle en masse; D The ischemia line is obvious after occlusion of hemihepatic pedicle

Table 2 Steps of building the liver hanging maneuver

\begin{tabular}{ll}
\hline Order & Steps \\
\hline (1) & Dividing the peritoneum on the right side of the IVC just inferior to the liver to expose the right adrenal gland \\
(2) & Dissecting the space from below upward between the hepatic parenchyma and the anterior and superior edge \\
of the right adrenal gland, and then along the right side of the IVC. Then the retrohepatic space is dissected \\
at the right of right hepatic vein (RHV) \\
Inserting a cylindrical cambered Goldfinger Dissector passing through the retrohepatic space and arriving at \\
the right side of the suprahepatic IVC \\
(3) \\
(4) tape was pulled through the retrohepatic tunnel for hanging the liver
\end{tabular}

\section{Result}

The patients consisted of 23 men and 6 women. Their median age was $51.8 \pm 10.6$ years (range 29-73). The
Child-Pugh level was Child-Pugh A in 28 patients and Child-Pugh B in one patient. The ICG R15 result of all the 29 patients was less than $15 \%$. Liver cirrhosis was found in 20 patients (19 due to hepatitis B virus and one due to hepatitis $\mathrm{C}$ virus). Another two patients had a 


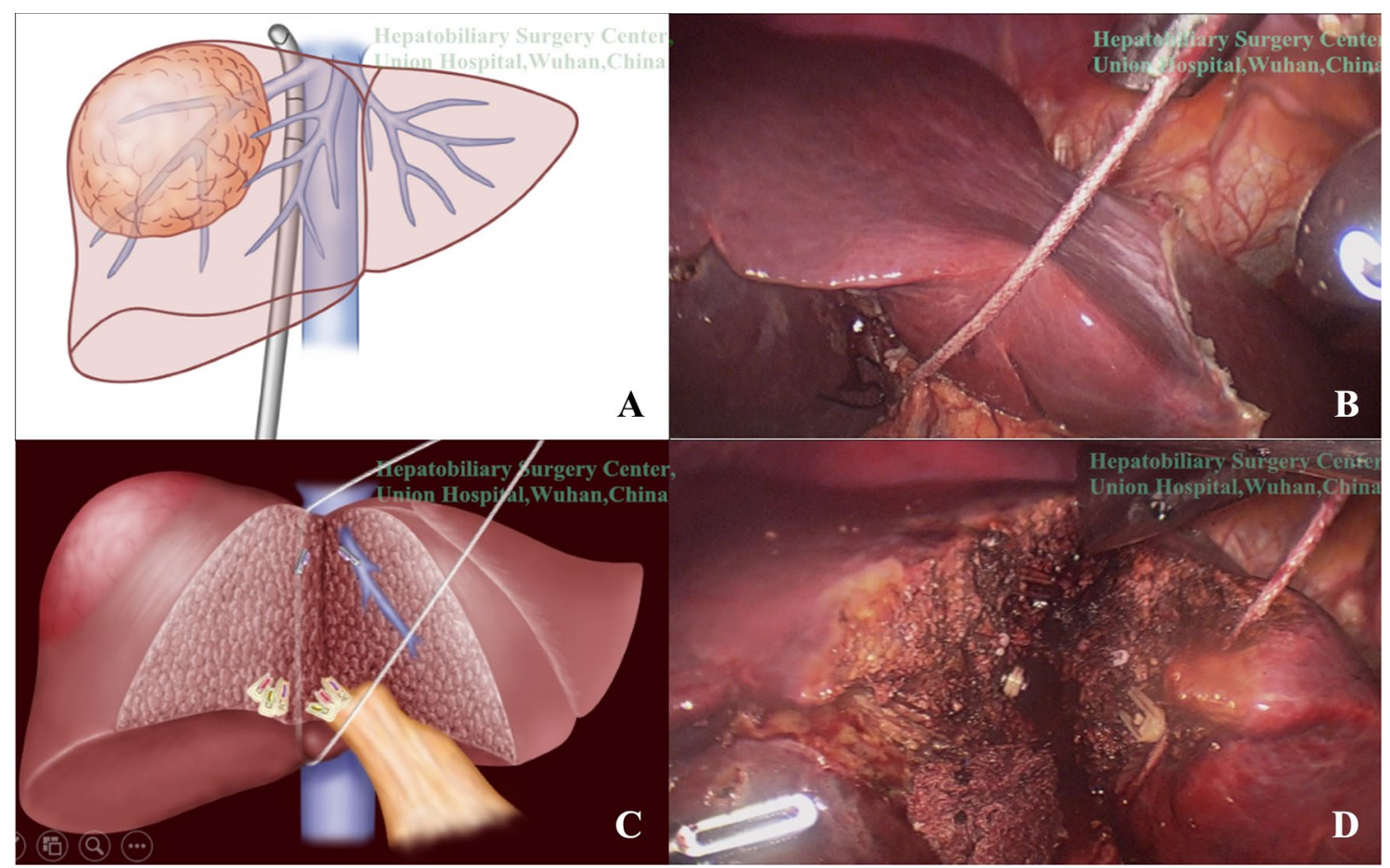

Fig. 3 Building the liver hanging maneuver. A The Goldfinger Dissector passing through the retrohepatic space along the right side of the suprahepatic IVC and penetrating out from the right side of RHV;
B A tape was pulled through the retrohepatic tunnel for hanging the liver. C, D The liver transection is guided by the hanging tape positive hepatitis B virus finding without liver cirrhosis. The histological diagnosis was hepatocellular carcinoma in 23 patients, intrahepatic cholangiocarcinoma in four patients, and hepatic metastasis of colonic carcinoma in two patients. The time of the first postoperative flatus was $2.10 \pm 0.96$ days. The details are shown in Table 3 .

All the 29 operations were successfully performed laparoscopically. The tumor size was $12.4 \pm 1.9 \mathrm{~cm}$, and the resection margin was $20.1 \pm 10.8 \mathrm{~mm}$. The operation time for LRH was $190.3 \pm 49.9 \mathrm{~min}$. The duration of RHP ligation was $10.0 \pm 3.0 \mathrm{~min}$, and that of laparoscopic LHM was $30.8 \pm 10.3 \mathrm{~min}$. The intraoperative blood loss volume during LRH was $281.7 \pm 117.8 \mathrm{~mL}$. The success rate of retrohepatic tunnel dissection and hemihepatic pedicle occlusion was $100 \%$. Five patients required blood transfusion, and the amount of blood transfusion was $300.0 \pm 89.4 \mathrm{~mL}$. The details are shown in Table 4.

The complication rate was $17.2 \%$. Pleural effusion combined with ascites occurred in one patient who was treated with thoracentesis for drainage (grade IIIa). Another pleural effusion in one patient was managed without surgical intervention (grade I). One patient had pneumonia, and two had refractory ascites. All of them were treated with pharmacological treatment. No postoperative bile leak occurred (grade II). The average hospital stay after operation was $10.0 \pm 2.9$ days. The perioperative mortality rate was 0 . The details are shown in Table 5 .

\section{Discussion}

Laparoscopy has become widely used in surgical operations owing to the microincision, less trauma, less pain, and quick recovery. Reports regarding LLR increased, such as LLRs for benign and malignant tumors, hepatolithiasis, hepatic cyst, and even associating liver partitioning and portal vein occlusion for staged hepatectomy (ALPPS) and living donor liver transplantation [6, 11-16], with the invention of different instruments, application of new techniques, modification of conventional methods, etc. However, complex LLRs, such as LRH, still remain to be explored because of the difficulty associated with bleeding control and exposure during laparoscopy.

Vascular occlusion is crucial for hepatectomy. Pringle was the first to advocate ligating the inflow vessels of the liver during transection of the parenchyma for bleeding reduction $[17,18]$. While ischemic damage is a major problem [19, 20], ligation and division of the corresponding vessels and ducts via dissection of the hepatic hilus for blood loss reduction during hepatic parenchyma transection when 
Table 3 Patient demographics and clinical data $(n=29)$

\begin{tabular}{|c|c|}
\hline Characteristics & Value \\
\hline Age (years) & $51.8 \pm 10.6(29-73)$ \\
\hline Sex ratio (M:F) & $23: 6$ \\
\hline \multicolumn{2}{|l|}{ Child-Pugh grade } \\
\hline A & $28(97 \%)$ \\
\hline B & $1(3 \%)$ \\
\hline \multicolumn{2}{|l|}{ Cirrhosis } \\
\hline Yes & $20(69 \%)$ \\
\hline No & $9(31 \%)$ \\
\hline \multicolumn{2}{|l|}{ Preoperative laboratory examinations } \\
\hline HBsAg positive & $22(76 \%)$ \\
\hline Anti-HCV positive & $1(3 \%)$ \\
\hline AFP positive & $16(55 \%)$ \\
\hline CEA positive & $3(10 \%)$ \\
\hline CA19-9 positive & $4(14 \%)$ \\
\hline CA125 positive & $7(24 \%)$ \\
\hline Hemoglobin (g/L) & $128.9 \pm 21.7$ \\
\hline Total bilirubin $(\mu \mathrm{mol} / \mathrm{L})$ & $16.1 \pm 6.9$ \\
\hline ALT (U/L) & $54.2 \pm 54.3$ \\
\hline AST (U/L) & $57.9 \pm 48.2$ \\
\hline Prothrombin time (s) & $13.7 \pm 1.2$ \\
\hline ALB (g/L) & $39.3 \pm 6.0$ \\
\hline ICG-R15 (\%) & $5.8 \pm 2.6$ \\
\hline Remnant functional/standard liver volume (\%) & $47.7 \pm 4.3$ \\
\hline \multicolumn{2}{|l|}{ Histological diagnosis } \\
\hline $\mathrm{HCC}$ & $23(79 \%)$ \\
\hline IHC & $4(14 \%)$ \\
\hline HMCC & $2(7 \%)$ \\
\hline
\end{tabular}

Values are expressed as mean \pm SD or number (\%)

$H B s$ Ag hepatitis B surface antigen, Anti-HCV hepatitis c virus antibody, $A F P$ alpha fetoprotein, $C E A$ carcinoembryonic antigen, $C A 19$ 9 carbohydrate antigen 19-9, CA125 carbohydrate antigen $125, A L T$ alanine aminotransferase, AST aspartate aminotransferase, ICG-R15 indocyanine green retention rate at $15 \mathrm{~min}, H C C$ hepatocellular carcinoma, IHC intrahepatic cholangiocarcinoma, HMCC hepatic metastasis of colonic carcinoma

Table 4 Intraoperative data $(n=29)$

\begin{tabular}{ll}
\hline Characteristics & Value \\
\hline Duration of operation (min) & \\
Total & $190.3 \pm 49.9$ \\
Ligating RHP & $10.0 \pm 3.0$ \\
Laparoscopic LHM & $30.8 \pm 10.3$ \\
Blood loss (mL) & $281.7 \pm 117.8$ \\
Patients required transfusion & $5(17 \%)$ \\
Transfusion (mL) & $300.0 \pm 89.4$ \\
Conversion & 0 \\
Tumor size (cm) & $12.4 \pm 1.9$ \\
Resection margin $(\mathrm{mm})$ & $20.1 \pm 10.8$ \\
\hline
\end{tabular}

Values are expressed as mean \pm SD or number (\%)
Table 5 Postoperative data $(n=29)$

\begin{tabular}{ll}
\hline Characteristics & Value \\
\hline complication & $5(17.2 \%)$ \\
Clavien-Dindo classification & \\
Grade I & 1 \\
$\quad$ Pleural effusion & \\
Grade II & 1 \\
$\quad$ Pneumonia & 2 \\
$\quad$ Ascites & \\
Grade IIIa & 1 \\
$\quad$ Pleural effusion & 0 \\
Reoperation & $2.10 \pm 0.96$ \\
Postoperative time to first flatus (days) & $10.0 \pm 2.9$ \\
Hospital stay (days) & 0 \\
Mortality &
\end{tabular}

Values are expressed as mean $\pm \mathrm{SD}$ or number (\%). Complications graded according to Clavien-Dindo classification

performing right hepatectomy were first described by LortatJacobs and Robert in 1952, which were known as anatomical hepatectomy or classical hepatectomy later [21]. However, the risk of hemorrhage during dissection and bile leaks occurring in the postoperative period makes this approach not suitable for laparoscopy. Chen and colleagues devised a simple vascular occlusion technique without tedious and time-consuming hilus dissection during open left and right hepatectomies [7]. By applying this technique for LLRs using a Goldfinger Dissector instead of a clamp, we obtained a satisfying result. We did not need to dissect the Glisson's sheath of the pedicle to ligate the artery, vein, and bile duct. We can control them en masse while avoiding bleeding and injuring the bile ducts during hilus dissection during LRH. It was convenient to perform such from our practice. The duration of the RHP ligation was only $10.0 \pm 3.0$ min during our operations. The feature of amplifying the view offered by endoscopes can help the operator ligate the hemihepatic pedicle.

In 2001, Belghiti et al. reported a kind of LHM for an easier parenchymal transection at deeper sites, better bleeding control, and shorter duration of transection using a tape to pass through the retrohepatic tunnel between the anterior surface of the IVC and the liver [9, 10]. Since then, LHM has been applied in various anatomical hepatectomy procedures worldwide, even including laparoscopic hepatectomy [10, 22-24]. Several other groups demonstrated a success rate of $94 \%$ of dissecting the retrohepatic tunnel blindly $[25,26]$. The rate of bleeding during retrohepatic dissection due to an injuring short hepatic vein was $4-6 \%$ as reported [27, 28]. Chen et al. modified the hanging maneuver in open right hepatectomy [8]. From our practice, we found that Chen's approach is 
very suitable for LLRs. First, the retrohepatic tunnel dissected at the right side of the IVC is avascular, which is the crucial difference from Belghiti's approach. Studying the anatomy of the liver, we can find that the retrohepatic right lateral IVC region consists of connective tissues, and the entire caudate lobe is located at the left side of this tunnel. In other words, this area is a part of the bare area of liver at the right side of IVC, which is avascular. The risk of bleeding during establishment of the tunnel is very low. However, the tunnel in Belghiti's approach passes through the anterior surface of the IVC. The short hepatic veins of the third porta of the liver, especially those drained from the caudate lobe, existed in this area $[29,30]$. The risk of injuring the IVC and its branches increased. The tapes pass through the right side of the right hepatic vein (RHV) instead of between the RHV and middle hepatic vein (MHV) in Belghiti's approach, so that there is less possibility to injure the RHV and MHV during dissection. Second, the tunnel was established via blunt dissection using the Goldfinger Dissector, which guaranteed lesser bleeding risks. Third, the important point is that we can have a better view at the retrohepatic space using the laparoscope during hanging, while tunnel dissection is performed blindly owing to a poor retrohepatic view in open surgery. During laparoscopic surgery, we can approximately visualize the tunnel (Fig. 3). The duration of this process was $30.8 \pm 10.3 \mathrm{~min}$. Further, there was no severe bleeding during our tunnel development. However, our suggestion for those willing to use this method is that they should avoid positioning the tunnel incorrectly. The right adrenal veins may be injured if the tunnel is established at too later position, which may lead to severe bleeding.

Based on these results, all the 29 operations were performed successfully using laparoscope without conversion. Only five patients needed transfusion $(300.0 \pm 89.4 \mathrm{~mL})$. Further, the duration of the two processes was short. No bile leakage occurred, since the risk of bile duct injury was avoided with these approaches. Two patients suffered refractory ascites. The main reason may be that the FLV/ SLV was too low (40.5 and 41\%).

In conclusion, LRH can also be performed feasibly, easily, and safely via the application of this new LHM and the simple vascular occlusion technique, owing to the satisfying outcomes of short operation time, less blood loss, and microincision, consequently providing good recovery. We believe that LRH may also be regarded as a standard treatment option with the application of Chen's methods.

\section{Compliance with ethical standards}

Disclosures Hongpeng Chu, Guojun Cao, Yong Tang, Xiaolong Du, Xiaobo Min, and Chidan Wan have no conflict of interests or financial ties to disclose.
Open Access This article is distributed under the terms of the Creative Commons Attribution 4.0 International License (http://creativecommons.org/licenses/by/4.0/), which permits unrestricted use, distribution, and reproduction in any medium, provided you give appropriate credit to the original author(s) and the source, provide a link to the Creative Commons license, and indicate if changes were made.

\section{References}

1. Xiang L, Li J, Chen J, Wang X, Guo P, Fan Y et al (2016) Prospective cohort study of laparoscopic and open hepatectomy for hepatocellular carcinoma. Br J Surg 103(13):1895-1901

2. Parks KR, Kuo YH, Davis JM, O'Brien B, Hagopian EJ (2013) Laparoscopic versus open liver resection: a meta-analysis of long-term outcome. HPB 16(2):109-118

3. Schiffman SC, Kim KH, Tsung A, Marsh JW, Geller DA (2015) Laparoscopic versus open liver resection for metastatic colorectal cancer: a metaanalysis of 610 patients. Surgery 157(2):211-222

4. Wakabayashi G, Cherqui D, Geller DA, Buell JF, Kaneko H, Han HS et al (2015) Recommendations for laparoscopic liver resection: a report from the second international consensus conference held in Morioka. Ann Surg 261(4):619-629

5. Buell JF, Cherqui D, Geller DA, O'Rourke N, Iannitti D, Dagher I et al (2009) The international position on laparoscopic liver surgery: the Louisville Statement, 2008. Ann Surg 250(5):825-830

6. Belli G, Gayet B, Han HS, Wakabayashi G, Kim KH, Cannon R et al (2013) Laparoscopic left hemihepatectomy a consideration for acceptance as standard of care. Surg Endosc 27(8):2721-2726

7. Chen XP, Zhang ZW, Huang ZY, Chen YF, Zhang WG, Qiu FZ (2011) Alternative management of anatomical right hemihepatectomy using ligation of inflow and outflow vessels without hilus dissection. J Gastroenterol Hepatol 26(4):663-668

8. Chen XP, Zhang WG, Lau WY, Qiu FZ (2008) Right hepatectomy using the liver double-hanging maneuver through the retrohepatic avascular tunnel on the right of the inferior vena cava. Surgery 144(5):830-833

9. Belghiti J, Guevara OA, Noun R, Saldinger PF, Kianmanesh R (2001) Liver hanging maneuver: a safe approach to right hepatectomy without liver mobilization. J Am Coll Surg 193(1):109-111

10. Dokmak S, Ben Safta Y, Ftériche FS, Aussilhou B, Belghiti J (2014) Pure laparoscopic right hepatectomy with the hanging maneuver for multiple hepatocellular adenomas. Ann Surg Oncol 21(12):3800-3801

11. Lin NC, Nitta H, Wakabayashi G (2013) Laparoscopic major hepatectomy: a systematic literature review and comparison of 3 techniques. Ann Surg 257(2):205-213

12. Yoon YS, Han HS, Cho JY, Kim JH, Kwon Y (2013) Laparoscopic liver resection for centrally located tumors close to the hilum, major hepatic veins, or inferior vena cava. Surgery 153(4):502-509

13. Han HS, Cho JY, Yoon YS, Hwang DW, Kim YK, Shin HK et al (2015) Total laparoscopic living donor right hepatectomy. Surg Endosc 29(1): 184

14. Takahara T, Wakabayashi G, Nitta H, Hasegawa Y, Katagiri $\mathrm{H}$, Umemura A et al (2017) The first comparative study of the perioperative outcomes between pure laparoscopic donor hepatectomy and laparoscopy-assisted donor hepatectomy in a single institution. Transplantation. https://doi.org/10.1097/ TP.0000000000001675

15. Xu J, Hu C, Cao HL, Zhang ML, Ye S, Zheng SS et al (2016) Meta-analysis of laparoscopic versus open hepatectomy for live liver donors. PLoS ONE 11(10):e0165319 
16. Xiao L, Li JW, Zheng SG (2015) Totally laparoscopic ALPPS in the treatment of cirrhotic hepatocellular carcinoma. Surg Endosc 29(9):2800

17. Pringle JH (1908) V. Notes on the arrest of hepatic hemorrhage due to trauma. Ann Surg 48(4):541

18. Zhang W, Wang J, Li C, Zhang Z, Dirie NI, Dong H et al (2017) Infrahepatic inferior vena cava clamping with Pringle maneuvers for laparoscopic extracapsular enucleation of giant liver hemangiomas. Surg Endosc. https://doi.org/10.1007/s00464-016-5396-6

19. Smyrniotis V, Kostopanagiotou G, Lolis E, Theodoraki K, Farantos C, Andreadou I et al (2003) Effects of hepatovenous back flow on ischemic- reperfusion injuries in liver resections with the Pringle maneuver. J Am Coll Surg 197(6):949-954

20. Cho JY, Han HS, Choi Y, Yoon YS, Kim S, Choi JK et al (2017) Association of remnant liver ischemia with early recurrence and poor survival after liver resection in patients with hepatocellular carcinoma. JAMA Surg. https://doi.org/10.1001/ jamasurg.2016.5040

21. Lortat-Jacob JL. Robert HG (1952) Well defined technic for right hepatectomy. Presse Med 60(26):549-551

22. Kim JH, Ryu DH, Jang LC, Choi JW (2016) Lateral approach liver hanging maneuver in laparoscopic anatomical liver resections. Surg Endosc 30(8):3611-3617

23. Troisi RI, Montalti R (2012) Modified hanging maneuver using the Goldfinger Dissector in laparoscopic right and left hepatectomy. Dig Surg 29(6):463-467
24. Nanashima A, Nagayasu T (2016) Development and clinical usefulness of the liver hanging maneuver in various anatomical hepatectomy procedures. Surg Today 46(4):398-404

25. Ogata S, Belghiti J, Varma D, Sommacale D, Maeda A, Dondero $F$ et al (2007) Two hundred liver hanging maneuvers for major hepatectomy: a single-center experience. Ann Surg 245(1):31-35

26. Gaujoux S, Douard R, Ettorre GM, Delmas V, Chevallier JM, Cugnenc PH (2007) Liver hanging maneuver: an anatomic and clinical review. Am J Surg 193(4):488-492

27. Kokudo N, Imamura H, Sano K, Zhang K, Hasegawa K, Sugawara $\mathrm{Y}$ et al (2005) Ultrasonically assisted retrohepatic dissection for a liver hanging maneuver. Ann Surg 242(5):651-654

28. Meng WC1, Shao CX, Mak KL, Lau PY, Yeung YP, Yip AW (2003) Anatomical justification of Belghiti's 'liver hanging manoeuvre' in right hepatectomy with anterior approach. ANZ J Surg 73(6):407-409

29. Chang RW, Shan-Quan S, Yen WW (1989) An applied anatomical study of the ostia venae hepaticae and the retrohepatic segment of the inferior vena cava. J Anat 164:41-47

30. Camargo AMSR., Teixeira GG, Ortale JR (1996) Anatomy of the ostia venae hepaticae and the retrohepatic segment of the inferior vena cava. J Anat 188:59-64 